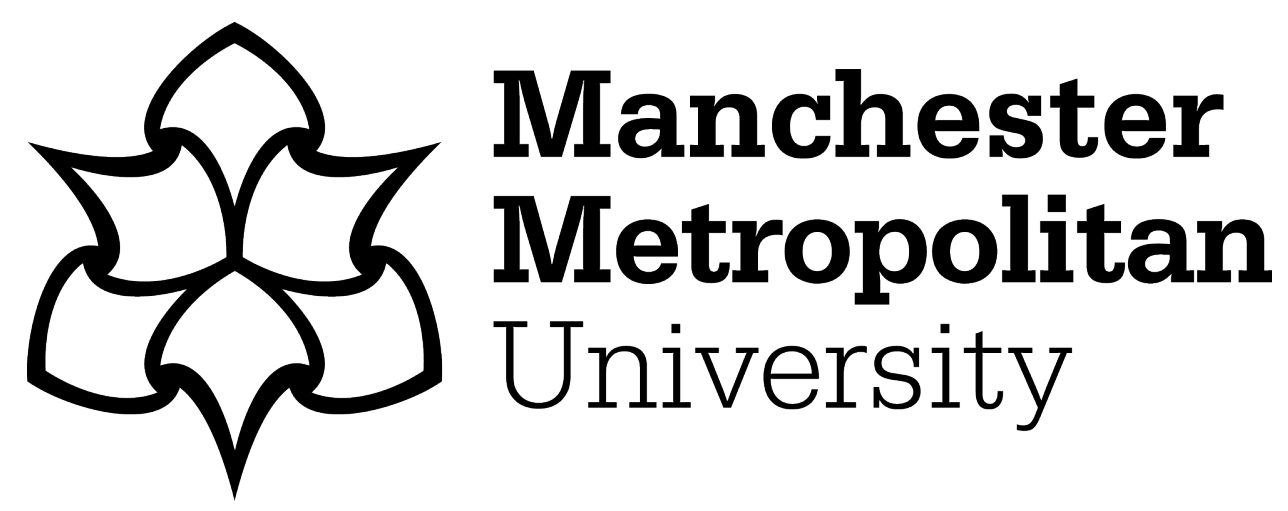

Hogarth, L, Nicholson, V, Spathis, J, Tweedy, S, Beckman, E, Connick, M, van de Vliet, P, Payton, C and Burkett, B (2018) A battery of strength tests for evidence-based classification in Para swimming. Journal of Sports Sciences, 37 (4). pp. 404-413. ISSN 0264-0414

Downloaded from: https://e-space.mmu.ac.uk/621732/

Version: Accepted Version

Publisher: Taylor \& Francis

DOI: https://doi.org/10.1080/02640414.2018.1504606

Please cite the published version 


\section{A BATTERY OF STRENGTH TESTS FOR EVIDENCE-BASED CLASSIFICATION IN PARA SWIMMING}

Luke Hogarth ${ }^{1}$, Vaughan Nicholson ${ }^{2}$, Jemima Spathis ${ }^{3}$, Sean Tweedy ${ }^{4}$, Emma Beckman ${ }^{4}$, Mark Connick ${ }^{4}$, Peter Van de Vliet ${ }^{5}$, Carl Payton ${ }^{6}$, Brendan Burkett ${ }^{1}$.

\footnotetext{
${ }^{1}$ School of Health and Sport Sciences, University of the Sunshine Coast, Sippy Downs, Australia.

${ }^{2}$ School of Physiotherapy, Australian Catholic University, Brisbane, Australia.

${ }^{3}$ School of Exercise Science, Australian Catholic University, Brisbane, Australia.

${ }^{4}$ School of Human Movement and Nutrition Sciences, University of Queensland, St Lucia, Queensland, Australia.

${ }^{5}$ Medical and Scientific Department, International Paralympic Committee, Bonn, Germany. ${ }^{6}$ HEAL Research Centre, Manchester Metropolitan University, Crewe, United Kingdom.
}

Running title: Strength impairment in Para swimming

Key words: disability, Paralympics, cerebral palsy, spinal cord injury, validity, reliability.

$\begin{array}{ll}\text { Abstract word count: } & 200 \\ \text { Text-only word count: } & 5018 \\ \text { Tables and/or figures: } & 5 \\ \text { Supplementary tables: } & 2\end{array}$

\section{Corresponding Author:}

Luke Hogarth

School of Health and Sport Sciences, University of the Sunshine Coast, Sippy Downs, Queensland, Australia

Postal address: Locked Bag 4, Maroochydore DC QLD 4558

Telephone: +61 754565065

Email: Ihogarth@usc.edu.au

\section{Acknowledgements:}

The authors would like to thank the participants for volunteering their time for this study. Also, the authors would like to acknowledge Jayden Lowrie, Samantha Yardy-Phelan, Hannah Jarvis and Ana Maia for their assistance in data collection. This research was supported by the International Paralympic Committee (IPC), UK Sport, Exercise \& Sports Science Australia (Applied Sports Science Research Grant), and the University of the Sunshine Coast (Faculty Research Initiatives Grants Scheme). Mark Connick, Emma Beckman and Sean Tweedy are members of the IPC Classification Research and Development Centre (Physical Impairments), which is supported by the International Paralympic Committee. 


\section{ABSTRACT}

4 This study examined the validity of isometric strength tests for evidence-based classification

5 in Para swimming. Thirty non-disabled participants and forty-two Para swimmers with

6 physical impairment completed an isometric strength test battery designed to explain

7 activity limitation in the freestyle discipline. Measures pertaining to dominant and non-

8 dominant limb strength and symmetry were derived from four strength tests that were

9 found to be reliable in a cohort of non-disabled participants (ICC $=0.85-0.97 ; \mathrm{CV}=6.4-9.1 \%$ ).

10 Para swimmers had lower scores in strength tests compared with non-disabled participants

$11 \quad(d=0.14-1.00)$ and the strength test battery successfully classified $95 \%$ of Para swimmers

12 with physical impairment using random forest algorithm. Most of the strength measures had

13 low to moderate correlations $(r=0.32$ to $0.53 ; p \leq 0.05)$ with maximal freestyle swim speed

14 in the cohort of para swimmers. Although, fewer correlations were found for both groups

15 when Para swimmers with hypertonia or impaired muscle power were analysed

16 independently, highlighting the impairment-specific nature of activity limitation in Para

17 swimming. Collectively, the strength test battery has utility in Para swimming classification

18 to infer loss of strength in Para swimmers, guide minimum eligibility criteria, and to define

19 the impact that strength impairment has on Para swimming performance. 
21 Classification plays an integral role in Paralympic sport and aims to promote increased

22 participation in sport by people with disabilities by minimising the impact that impairment

23 has on the competition outcome. Para swimming, one of the most popular Paralympic sports,

24 uses a functional classification system to group athletes with an eligible physical impairment.

25 Unfortunately, studies have shown current classification methods fail to delineate

26 performance between some classes and disadvantage athletes with certain types of physical

27 impairment within classes (Burkett et al., 2018; Daly \& Vanlandewijck, 1999; Wu \& Williams,

28 1999). The shortcomings of the current classification system may result, at least in part, from

29 issues with measurement weighting and aggregation stemming from a lack of understanding

30 of the relationship between impairment and swimming performance (Tweedy, Beckman, \&

31 Connick, 2014). World Para swimming have mandated that research be conducted to provide

32 the scientific evidence to underpin a new classification system in Para swimming

33 (International Paralympic Committee, 2015).

34 A key step towards evidence-based classification systems in Para sport is developing valid

35 tests of impairment and establishing their relationship with sports performance. It is 36 important to note that these tests do not directly measure impairment, but infer impairment

37 based on knowledge of intact, unimpaired body structures and functions (Tweedy, Mann, \&

38 Vanlandewijck, 2016). Their purpose is to describe Para athletes' type, location and severity

39 of impairment to estimate their subsequent activity limitation for a given sporting event. The

40 International Paralympic Committee (IPC) Position Stand stipulates that valid impairment

41 tests will have several measurement properties (Tweedy \& Vanlandewijck, 2011). These

42 include impairment tests being precise and reliable, ratio-scaled, specific to the impairment

43 of interest, quantitative, account for a significant portion of variance in performance, and as

44 training resistant as possible. 
45 Muscular strength and power are key determinants of success in competitive swimming and 46 their importance to propulsion during swimming is widely accepted (Crowley, Harrison \& 47 Lyons, 2017; Loturco et al., 2016). Para swimmers with health conditions such as spinal cord 48 injury, cerebral palsy and Charcot-Marie-Tooth disease have impairments to the central and 49 peripheral nervous systems, musculoskeletal system or links between these structures, that 50 result in loss of muscular strength and power and affect their swimming performance 51 (Dingley, Pyne, \& Burkett, 2014; Dingley, Pyne, Youngson \& Burkett, 2015; Morouco et al., 52 2011). Classifying strength impairment of Para swimmers with motor-complete spinal cord 53 injury is relatively straightforward as these athletes have a non-progressive loss of voluntary 54 motor control that corresponds to the level of lesion (Connick et al., 2018). Other progressive 55 and non-progressive medical conditions such as cerebral palsy, motor-incomplete spinal cord 56 injury, polio, and Charcot-Marie-Tooth disease have inconsistent clinical manifestations. 57 Para swimmers with these conditions have loss of voluntary motor control that varies 58 considerably for the severity of impairment and its presentation in the trunk, and upper and 59 lower limb extremities.

60 Manual Muscle Testing (MMT) techniques are currently used to assess the severity and

61 location of impairment by subjectively inferring swimmers' loss of strength by rating whether 62 they can produce what is termed 'normal' resistance around joints (International Paralympic 63 Committee, 2017). Although having several advantages, including being easy to administer, 64 widely utilised in clinical practice and inexpensive, MMT techniques lack key measurement 65 properties required for evidence-based classification. Inter-and intra-tester reliability is poor 66 due to the subjective assessment of muscle strength and the ordinal measures derived from 67 MMT are limited in defining their relationship with sporting performance (Beckman, Connick, 68 \& Tweedy, 2017; Bohannon, 2005). 
69 Guidelines have recently been published for the development of instrumented tests of

70 muscle strength for the purposes of classification (Beckman, Connick \& Tweedy, 2017). The

71 key recommendations were to develop isometric measures of muscle strength that assess

72 Para athletes' force generating capacity in multi-joint positions that are standardised and

73 specific to the sport of interest. Such tests will provide the most valid measures for inferring

74 loss of muscle strength for classification as they determine the maximal force generating

75 capacity of a muscle or muscle group (Cormie, McGuigan, \& Newton, 2011), are more likely

76 to be resistant to training than dynamic muscular strength and power tests that typically

77 have greater specificity to athletic performance (Beckman et al., 2017; Loturco et al., 2016),

78 and might have strong and meaningful associations with sports performance in Para athletes

79 with strength impairment (Beckman, Conncik \& Tweedy, 2016; Hyde et al., 2017).

80 As isometric strength tests are limited in assessing muscular strength through full range of

81 motion, important steps in developing tests for classification include identifying the principal

82 muscle groups and actions that are involved in the sport (Beckman et al., 2017; Burkett et al.,

83 2017). Most studies in able-bodied swimmers have investigated front crawl swimming and

84 have reported the latissimus dorsi, pectoralis major, and teres minor play important roles in

85 stabilising and mobilising the shoulder into extension and adduction during the early and late

86 underwater pull phases that are primarily responsible for propulsion (Amaro, Morouco,

87 Marques, Fernandes \& Marinho, 2017; Martens, Figueiredo \& Daly, 2015). Agonist

88 antagonist activity of muscles of the elbow joint (i.e. biceps brachii and triceps brachii) and

89 wrist joint (i.e. brachioradialis, flexor carpi ulnaris, and extensor carpi ulnaris) stabilise the

90 forearm and hand to overcome water drag during these propulsive actions (Martens et al.,

91 2015).

92 Although the lower limb extremity contributes less to propulsion and swim velocity in front

93 crawl than the upper limb extremity (Amaro et al., 2017; Bartolomeu, Costa \& Barbosa, 2018), 
94 the quadriceps and hamstring muscle groups mobilise the hip and knee joints to generate

95 drag and lift forces in coordination with the arm stroke (Bartolomeu et al., 2018; Martens et

96 al., 2015; Morouco, Marinho, Izquierdo, Neiva \& Marques, 2015). Strength impairments in

97 the lower limb extremity might have increased importance in the lower sport classes where

98 drag is more important in discriminating between performances (Oh, Burkett, Osborough,

99 Formosa \& Payton, 2013), due to the role that the leg kick plays in stabilising the body and

100 generating lift forces that allow swimmers to maintain streamlined body positions (Amaro et

101 al., 2017; Bartolomeu et al., 2018; Psycharakis \& Sanders, 2010). Lower body muscular

102 strength and power are also key determinants of starts and turns performance with the

103 gluteus maximus and triceps surae contributing to joint torque during hip extension and

104 plantar flexion, respectively, to generate propulsive actions during certain components of a

105 swim race (Jones, Pyne, Haff \& Newton, 2018; Morouco, Marinho, Amaro, Perez-Turpin \&

106 Marques, 2012).

107 This study presents isometric strength tests that have been designed to infer loss of muscular

108 strength in the upper and lower limb extremities for evidence-based Para swimming

109 classification. The aims were to: (i) examine the predictive validity of isometric strength tests

110 to discriminate between non-disabled participants and Para swimmers with physical

111 impairments, (ii) establish the strength of association between isometric strength tests and

112 freestyle swim performance in Para swimmers with strength impairments, and (iii) establish

113 the test-retest reliability of isometric strength tests in non-disabled participants. Isometric

114 strength tests might have utility in Para swimming classification if they discriminate Para

115 swimmers with strength impairment from non-disabled participants, have meaningful 116 associations with swimming performance in Para swimmers, and are found to be reliable. 


\section{Participants}

Data were collected from 72 participants including Para swimmers and non-disabled participants (Table 1). Para swimmers had an eligible physical impairment resulting in loss of muscle power. They had received national or international classification, were undertaking planned training regimes and competing at a national or international level. Non-disabled participants were recruited from University of the Sunshine Coast, Australia or Manchester Metropolitan University, United Kingdom. They were between the ages of 18 and 35 years of age, apparently healthy and recreationally active (undertaking planned exercise, training or sport at least twice a week for a minimum total of 80 minutes). These eligibility criteria were established to recruit a convenient sample of non-disabled participants with a wide range of activity backgrounds. Such a cohort was considered advantageous when examining the predictive validity of strength tests to identify participants with and without physical impairment. All participants gave their written informed consent to participate in this study under approved ethical guidelines $(\mathrm{A} / 16 / 892)$.

\section{Design}

Isometric strength tests were developed by the research team consisting of experts in evidence-based classification and Para swimming sport science. Tests were designed to explain activity limitation in the freestyle discipline. They went through a development process that included consultation with a panel of coaches, Para swimmers, classifiers, administrators and sport science and medicine personnel, and were piloted in individuals with disabilities. Para swimmers completed the test battery during organised data collection events within Europe and Australia. They completed physical impairment and swimmingspecific assessments around their training schedules during these events. Non-disabled participants and Para swimmers attended at least one 90-minute session where they undertook the finalised test battery comprising four strength tests. Para swimmers also 
144 attended a separate 30-minute session where their maximal freestyle swim performance 145 was assessed. Non-disabled participants were asked to maintain their usual exercise or 146 training regimes throughout their involvement in the study. Fifteen non-disabled 147 participants repeated the test battery within a week to examine the test-retest reliability of 148 strength tests.

149 Experimental procedures

150 Participants completed a questionnaire regarding demographics, their typical training 151 regime (type and frequency of training), and training activity on the day of testing. Para swimmers also provided information pertaining to their training experience, competition standard attained, current sport class, and type of physical impairment. These data were verified against information attained from classification records listed in the IPC Sports Data Management System (https://db.ipc-services.org/sdms). Participants' stature and body mass were recorded prior to the strength tests. Stature was estimated from sitting height recorded from a custom-built chair for Para swimmers with no or poor locomotor ability. Ratios of sitting height to standing height available in the World Para swimming Classification Manual were used for estimations (International Paralympic Committee, 2017).

The order of the strength tests was randomised. All participants undertook the test battery under the instruction and supervision of the principal researcher. Isometric strength was assessed using an S-type strain gauge attached to a custom-made aluminium frame that provided force-time data collected at $200 \mathrm{~Hz}$ (Ergotest, Porsgrunn, Norway). The strength test battery consisted of 4 tests that yielded 8 outcome measures: dominant and nondominant (i) shoulder extension strength, (ii) shoulder flexion strength, (iii) hip extension strength and (iv) hip flexion strength. The strength test protocols are outlined in detail in Supplementary Table 1. Following practice trials, participants performed 3 maximal effort trials for each test. Once in position, participants were instructed to slowly build up their 
applied force until reaching their maximal effort within 2-3 seconds. All contractions lasted between 4 and 10 seconds and were performed on each minute, giving participants at least 50 seconds rest between consecutive trials (Beckman, Newcombe, Vanlandewijck, Connick, \& Tweedy, 2014). Each participant was given the same set of instructions before and during contractions. The best trial indicated by the highest maximal voluntary contraction (MVC) was used for analysis. For each strength test a symmetry index was calculated as a ratio of their non-dominant to dominant limb strength.

Para swimmers maximal clean swim speed was assessed over a $10 \mathrm{~m}$ calibrated test zone for their preferred freestyle swim stroke. Clean swim speed was determined using standard twodimensional video analysis procedures. Output from a $50 \mathrm{~Hz}$ video camera (Sony HDR HC9, Sony Corporation, Japan) placed perpendicular to the swimmers' direction of travel was captured using commercial software (Dartfish TeamPro version 7.0, Dartfish UK). Participants were instructed to reach maximal swim speed prior to the start of the $10 \mathrm{~m}$ test zone and sustain maximal swim speed until $5 \mathrm{~m}$ past the end of the test zone. They performed two maximal effort trials separated by a minimum of 3 minutes' rest and the fastest time to cover the $10 \mathrm{~m}$ test zone was used to compute their maximal clean swim speed. The recorded maximal clean swim speeds were found to have strong relationships with personal best race times for $50 \mathrm{~m}$ freestyle $\left(R^{2}=0.914\right)$ and $100 \mathrm{~m}$ freestyle $\left(R^{2}=0.892\right)$ in our participant cohort. Maximal clean swim speed was not assessed for three Para swimmers with hypertonia due to limited time with these participants.

\section{Statistical analyses}

Statistics were calculated using R version 3.4.0 (R Core Team, 2017). Shapiro-Wilks tests indicated non-uniform distribution of several test measures for Para swimmers with hypertonia or impaired muscle power. A Kruskal-Wallis rank test was used to determine significant effects between hypertonia, impaired muscle power and non-disabled participant 
194 groups. Wilcoxon tests were used post hoc to determine the source of significant effects,

195 with p-values adjusted for multiple comparisons using the Benjamini and Hochberg method.

196 Cliff's Delta (d), a non-parametric measure of effect size, was calculated with 95\% confidence

197 intervals to indicate the magnitude of difference in strength test measures between Para

198 swimmers and non-disabled participants (Rogmann, 2013). Sex-specific differences were

199 calculated as there were significant differences found in isometric strength measures

200 between non-disabled male and female participants.

201 Random forest algorithm was used to establish the predictive validity of strength tests to 202 classify participants with and without strength impairment. Random forest is a non-linear 203 machine learning technique that uses an ensemble learning method for classification and 204 regression (Liaw \& Wiener, 2002; Woods, Veale, Fransen, Robertson \& Collier, 2018). 205 Separate models were built to determine the prediction accuracies based on sex. The 206 importance of predictor variables was determined using the mean decrease in accuracy, 207 which indicates the decrease in prediction accuracy that occurs when a single variable is 208 excluded during the out-of-bag error calculation (Liaw \& Wiener, 2002; Woods et al., 2018).

209 Spearman correlation coefficients were calculated to assess the strength of association 210 between the Para swimmers' strength test measures and maximal clean swim speeds. 211 Correlations were calculated for the entire cohort of Para swimmers and independently for 212 the hypertonia and impaired muscle power groups. Significance was set at an alpha value of $213 \leq 0.05$. The strength of correlations was interpreted as negligible (0.0-0.2), low (0.21-0.40), 214 moderate (0.41-0.60), high (0.61-0.80) and very high (>0.81) (Mukaka, 2012).

215 For non-disabled participants, normality of distribution was confirmed using the Shapiro216 Wilk test. Unpaired sample t-tests assuming equal variances were used to determine 217 differences between male and female participant groups. Reliability assessments were 218 calculated using Hopkins' reliability spreadsheet (Hopkins, 2015). Paired sample t-tests were 
conducted to identify any systematic change in test measures between repeated trials. Intraclass correlation coefficients (ICC) method 3,1, standard error of measurement (SEM) scores expressed in the original units of measurement, and coefficient of variation (CV) scores were calculated to provide an absolute assessment of reliability (Hopkins, 2000).

\section{RESULTS}

Differences in strength scores between Para swimmers and non-disabled participants are shown in Figure 1. Para swimmers showed significantly lower strength scores for all tests, except for shoulder flexion strength in female Para swimmers (Figure 1C and 1D) and dominant hip flexion strength in male Para swimmers with hypertonia (Figure 1G). Para swimmers showed larger differences in strength scores compared with non-disabled participants for their non-dominant limbs (Figure 1). This was illustrated in differences between non-disabled participants and Para swimmers for symmetry indexes calculated for shoulder extension strength (mean \pm range $=0.96 \pm 0.12$ versus $0.82 \pm 0.51 ; d=0.81, p<0.01$ ), shoulder flexion strength (mean \pm range $=0.94 \pm 0.14$ versus $0.84 \pm 0.55 ; d=0.52, p<0.01$ ), hip extension strength (mean \pm range $=0.94 \pm 0.16$ versus $0.48 \pm 0.99 ; d=0.77, p<0.01$ ), and hip flexion strength (mean \pm range $=0.95 \pm 0.11$ versus $0.49 \pm 0.97 ; d=0.89, p<0.01$ ).

Random forest that included all strength test measures as predictor variables successfully classified 25/26 (96 \%) male Para swimmers and 15/16 (94 \%) female Para swimmers. The mean decrease in accuracy scores were similar for the male and female participant groups, with lower limb strength and symmetry measures typically being the most important variables for prediction of participants with and without physical impairment (Figure 2).

Maximal clean swim speeds were $1.14 \pm 0.34 \mathrm{~m} . \mathrm{s}^{-1}$ (range 0.21 to $1.62 \mathrm{~m} . \mathrm{s}^{-1}$ ) for male Para swimmers and $1.03 \pm 0.29 \mathrm{~m} . \mathrm{s}^{-1}$ (range 0.55 to $1.51 \mathrm{~m} . \mathrm{s}^{-1}$ ) for female Para swimmers. Para 
swimmers with hypertonia $\left(1.19 \pm 0.27 \mathrm{~m} \cdot \mathrm{s}^{-1}\right.$; range 0.55 to $\left.1.62 \mathrm{~m} \cdot \mathrm{s}^{-1}\right)$ had slightly faster clean swim speeds than Para swimmers with Impaired muscle power $\left(1.00 \pm 0.35 \mathrm{~m} . \mathrm{s}^{-1}\right.$; range 0.21 to $\left.1.51 \mathrm{~m} \cdot \mathrm{s}^{-1}\right)$, although there was no significant difference found between groups $(p=0.12)$. All strength scores had significant low to moderate correlations ( $r=0.32$ to $0.53, p \leq 0.05$ ) with maximal clean swim speed in the combined cohort of Para swimmers, except for nondominant shoulder flexion $(r=0.15, p=0.35)$ (Figure 3).

There were fewer strength scores that had significant correlations with clean swim speeds when hypertonia or impaired muscle power groups were analysed independently (Figure 3). Dominant and non-dominant shoulder extension strength had the strongest correlations with maximal clean swim speed for Para swimmers with hypertonia ( $r=0.46$ to $0.66, p \leq 0.04$ ) and impaired muscle power ( $r=0.47$ to $0.51, p \leq 0.04)$. Para swimmers with hypertonia also showed significant correlations between clean swim speed and strength scores for dominant shoulder flexion $(r=0.66, p<0.01)$ and dominant hip flexion $(r=0.44, p=0.05)$, while there were no correlations found for other strength tests $(r=0.27$ to $0.38, p=0.10$ to 0.25$)$. Para swimmers with impaired muscle power reported no significant correlations between clean swim speed and strength scores for shoulder flexion ( $r=-0.12$ to $0.12, p=0.61$ to 0.63 ), hip extension ( $r=0.12$ to $0.31, p=0.20$ to 0.30 ), or hip flexion ( $r=0.12$ to $0.19, p=0.45$ to 0.61 ).

Reliability assessments indicated all strength tests to be reliable in non-disabled participants (Table 2). There were no significant changes in outcome measures between repeated trials, with participants' absolute and relative changes ranging from $-7 \pm 4 \mathrm{~N}$ to $2 \pm 18 \mathrm{~N}$ and $-5 \pm$ $10 \%$ to $3 \pm 12 \%$, respectively. Strength test measures in non-disabled participants are shown in Figure 1 and Supplementary Table 2. Unpaired sample t-tests assuming equal variances indicated significant differences $(p<0.01)$ between non-disabled male and female participants for all strength tests, except for measures of strength symmetry. 


\section{DISCUSSION}

This study aimed to establish the validity and reliability of isometric strength tests for classification of Para swimmers with physical impairment. A key measurement property of these tests is their ability to identify Para swimmers with an eligible strength impairment. Eligibility is determined by type of physical impairment, as well as impairment severity that must conform to the minimum eligibility criteria. The isometric strength tests presented in this study were found to differ between Para swimmers with physical impairments and nondisabled participants (Figure 1), suggesting they will be useful in inferring loss of strength and guiding minimum eligibility criteria in Para swimming cohorts.

The strength test measures reported for non-disabled participants provide a useful benchmark to infer loss of muscle strength in Para swimmers, although there are several points to consider beforehand. First, there were significant differences in strength scores between non-disabled male and female participants suggesting that sex-specific benchmarks should be used to infer loss of strength in Para swimmers with physical impairment (Supplementary Table 2). Second, the non-disabled participants showed considerable variations in strength scores (Figure 1), likely due to the range in reported activity backgrounds (Table 1). Given that muscular strength is responsive to training type, volume and intensity (Crowley et al., 2017) it is important that normative values are collected in a larger sample of able-bodied swimmers with various training ages and regimes. This will provide classifiers with normative values in non-disabled participants stratified by age, sex and training status so that they can accurately infer Para swimmers' strength impairments.

Supporting the predictive validity of the isometric strength test battery, the random forest algorithm had a $95 \%$ success rate in correctly classifying participants with and without physical impairment based on strength test measures. There were two Para swimmers that were incorrectly classified as non-disabled participants. The first was a male Para swimmer 
with hemiplegic cerebral palsy that competes in the $\mathbf{S} 6$ class based on classification of motor coordination impairment, and so it is possible that that this participant is not affected by strength impairment. For Para swimmers with hypertonia, the current classification system assigns class based on the assessment of strength, motor coordination or range of movement depending on which one of these is judged to be most affected by the Para swimmer's health condition (International Paralympic Committee, 2017). It is interesting that all Para swimmers with hypertonia in this study compete in their current sport class based on assessment of motor coordination impairment. The high success rate of the random forest in classifying these Para swimmers using isometric strength and symmetry scores indicates that these Para swimmers have strength impairments that affect their swimming performance (Figure 3). This finding highlights the complexity of these Para swimmers' health conditions, and that classification should collectively account for impairments in strength, motor coordination and range of motion for these swimmers.

The incorrect classification of the female Para swimmer by the random forest algorithm raises several questions of the isometric strength test battery. This Para swimmer has an incomplete L4-L5 spinal cord injury and competes in the S8 sport class at Paralympic and World Championship standard. The random forest algorithm assigned $40 \%$ of the votes to the priori case most likely as the participant's strength scores were within or higher than the lower and upper quartiles for scores in non-disabled females, except for dominant and nondominant hip extension. This highlights the requirement of obtaining normative values in highly trained able-bodied swimmers to accurately infer strength impairment. Further, based on their classification records the Para swimmer was most affected by limited strength around the ankle joint. Active ankle range of motion is important to effectively orientate the foot segment during leg kicking to generate drag and lift forces (Connaboy et al., 2016), and plantar flexion at the ankle joint contributes to propulsion during starts and turns (Jones et al., 2018; Morouco et al., 2012). Although active range of motion assessments might explain 
part of this swimmer's activity limitation (Nicholson et al., 2018), these results indicate that the isometric strength test battery is not entirely comprehensive.

321 An important aspect of this study was examining the convergent validity of isometric

322 strength tests by establishing their strength of association with freestyle swim performance.

323 When the entire para swimming cohort was included in analyses there were low to moderate

324 correlations found between maximal swim speed and all isometric strength scores, except

325 for non-dominant shoulder flexion (Figure 3). Para swimmers showed the strongest 326 correlations between isometric shoulder extension strength and maximal clean swim speed

327 (Figure $3 \mathrm{~A}$ and $3 \mathrm{~B}$ ). The upper limb extremity contributes to most of the propulsive force 328 during tethered front crawl swimming (Amaro et al., 2017; Morouco et al., 2015), and the shoulder position during this test represented the start of the underwater push phase where able-bodied swimmers achieve the highest absolute hand speeds (Samson, Monnet, Bernard, Lacouture \& David, 2015). The lower limb extremity contributes less to propulsion in front crawl swimming (Amaro et al., 2017; Morouco et al., 2015), which explains the lower correlations found between hip flexion and extension strength and freestyle swim

334 performance in the combined cohort of Para swimmers (Figure 3). The leg kick is important 335 in stabilising and controlling body roll in coordination with the arm stroke (Bartolomeu et al., 336 2018; Psycharakis \& Sanders, 2010) and generates drag and lift forces that are likely to have 337 higher contributions to instantaneous swim velocity in cases where the arm stroke is limited 338 by impairment (Morouco et al., 2015; Bartolomeu et al., 2018). However, these tests might 339 not comprehensively describe knee flexion and plantar flexion strength impairments that 340 relate to starts and turns performance (Dingley et al., 2015; Jones et al., 2018) or propulsive 341 forces during swim kicking (Connaboy et al., 2016).

342 Ensuring that the isometric strength test battery is comprehensive and parsimonious is 343 important to consider before its implementation into a revised classification system. It is 
344 important to highlight that there were fewer correlations found between strength scores

345 and maximal swim speeds when hypertonia and impaired muscle power groups were

346 analysed independently (Figure 3). There are two explanations for these results. First, the

347 wide range in location and distribution of strength impairment of Para swimmers that are

348 within these groups affect the ability of any singular strength score to explain activity

349 limitation in swimming. For instance, Para swimmers with impaired muscle power had a

350 range of medical conditions (Table 1), some that might cause an even distribution of strength

351 impairment across the upper and lower limbs (e.g. Charcot-Marie-Tooth disease) and others

352 where strength impairment is confined to the trunk and lower limbs (e.g. complete $\mathrm{SCl}$ ).

353 Despite no correlation being found between lower limb strength and swim performance

354 within this group (Figure 3), lower limb strength scores might be useful in explaining activity

355 limitation in Para swimmers that have some remaining lower limb muscle power due to the

356 role of leg kick in controlling body roll and stabilising the torso (Bartolomeu et al., 2018;

357 Psycharakis \& Sanders, 2010). Conversely, the assessment of trunk impairment might be

358 more important in understanding activity limitation in Para swimmers with complete $\mathrm{SCl}$ that

359 cannot leg kick due to having no lower limb muscle power (Altman et al., 2017; Altman et al.,

360 2018; Psycharakis \& Sanders, 2010).

361 Another explanation for the above, is that the type of physical impairment influences the

362 association between strength tests and para swimming performance. It is interesting to note

363 that Para swimmers with hypertonia showed a high correlation between dominant shoulder

364 flexion and maximal clean swim speed $(r=0.66, p<0.01)$, while there was no correlation found

365 in Para swimmers with impaired muscle power (Figure 3C). This test was included in the

366 battery as it was thought it would describe activity limitation in Para swimmers with severe

367 impairments that use modified swim strokes (Prins \& Murata, 2008). The positioning and

368 action of the isometric shoulder flexion test is dissimilar to the kinematics of the underwater

369 and recovery stroke phases of front crawl in able-bodied swimmers (Martens et al., 2015), 
370 which explains why no correlation was found with maximal swim speed in the impaired

371 muscle power group. Conversely, the shoulder flexion strength test might be associated with

372 the level of spasticity that affects Para swimmers with hypertonia and may be collinear with

373 reduced motor coordination and range of motion that affects swim performance. Indeed,

374 spasticity typically affects the flexor, adductor and internal rotator muscle groups more than

375 their antagonists (Antunes, Rossato, Lima Kons, Luiz Sakugawa \& Fischer, 2017; Delgado \&

376 Albright, 2003), and there is a high inverse association between the level of spasticity and

377 voluntary motor function in people with health conditions such cerebral palsy and acquired

378 brain injury (Delgado \& Albright, 2003). These results highlight the impairment-specific

379 nature of activity limitation in Para swimming, and that separate test batteries could be used

380 to classify Para swimmers based on their aetiology of impairment.

381 The final aim of this study was to establish the test-retest reliability of strength tests. All tests

382 were shown to be reliable in non-disabled participants, which is a prerequisite for evidence-

383 based classification. Unfortunately, reliability in Para swimmers with hypertonia or impaired

384 muscle power was not assessed due to limited time available to test these swimmers.

385 Reliability data was collected in a convenient sample of non-disabled participants as

386 measures that were found to be unreliable in this cohort would be unlikely to have

387 acceptable reliability in Para swimmers with physical impairments (Beckman et al., 2014;

388 Connick, Beckman, Deuble \& Tweedy, 2016; Nicholson et al., 2018). Future studies should

389 now establish the reliability of measures in Para swimmers with physical impairments to

390 confirm their utility in Para swimming classification.

391 It is important to note that the application of this study's findings is limited without further

392 research. This study intentionally limited tests that were designed to explain activity

393 limitation in the freestyle discipline as there was limited time available to test Para swimmers.

394 While there is likely to be some crossover between tests, other swim strokes are dependent 
on muscle groups and actions that were not assessed in this study (Martens et al., 2015).

Targeted efforts are now required to develop strength tests that explain activity limitation in other swim strokes. Once this has been achieved, data collection in a larger sample of para swimmers can be conducted to define the relative impact that strength impairments have on swimming performance and guide valid classification structures (Altman et al., 2018; Connick et al., 2018; Hogarth, Payton, Van de Vliet, Connick \& Burkett, 2018).

401 The isometric strength tests in this study also have several inherent limitations in classifying 402 strength impairment. Namely, they are susceptible to athletes misrepresenting their abilities, 403 they limit strength assessment to a fixed range of motion, strength scores might be susceptible to fatigue induced by prior activity or the tests themselves, and measures might be responsive to sport-specific training regimes (McGuigan, Newton, Winchester \& Nelson, 2010). Even with these limitations, the objective measurement of strength impairment will undoubtedly improve the accuracy and transparency of Para swimming classification compared with current methods (Connick et al., 2018). Additionally, longitudinal assessments of isometric strength in Para swimmers will provide insights into their responsiveness to sport-specific training regimes so that classifiers can more accurately infer

411 strength impairment, and machine learning algorithms can predict competitive 412 performances from objective impairment measures to identify outlying performances 413 caused by intentional misrepresentation of abilities (Hogarth et al., 2018).

\section{CONCLUSIONS}

This study presented isometric strength tests that were developed to permit evidence-based

417 classification in Para swimming. Strength test measures had acceptable reliability in non-

418 disabled participants - a requisite of evidence-based classification. Differences in strength

419 test measures were found between non-disabled participants and Para swimmers with 
420 hypertonia or impaired muscle power, and random forest algorithm successfully classified

$42195 \%$ of Para swimmers. These results indicate that these tests will be useful in inferring loss

422 of strength in Para swimmers with strength impairment and guiding minimum eligibility

423 criteria. Dominant and non-dominant strength scores also had significant correlations with

424 maximal freestyle swim speed in Para swimmers. This suggests that strength tests will be

425 useful in explaining activity limitation in Para swimming, although results indicate that the

426 type and aetiology of physical impairment influence the utility of some strength tests.

427 Collectively, the results of this study make a significant contribution toward evidence-based

428 methods of classification for Para swimmers with strength impairments. 


\section{REFERENCES}

Altmann, V. C., Groen, B. E., Hart, A. L., Vanlandewijck, Y. C., van Limbeek, J., \& Keijsers, N. L. W. (2017). The impact of trunk impairment on performance-determining activities in wheelchair rugby. Scand J Med Sci Sports, 27(9), 1005-1014. doi: $10.1111 / \mathrm{sms} .12720$

Altmann, V. C., Groen, B. E., Hart, A. L., Vanlandewijck, Y. C., \& Keijsers, N. L. W. (2018). Classifying trunk strength impairment according to the activity limitation caused in wheelchair rugby performance. Scand J Med Sci Sports, 28(2), 649-657. doi: $10.1111 /$ sms.12921

Amaro, N. M., Morouco, P. G., Marques, M. C., Fernandes, R. J., \& Marinho, D. A. (2017). Biomechanical and bioenergetical evaluation of swimmers using fully-tethered swimming: A qualitative review. Journal of Human Sport and Exercise, 12(4), 13461360. doi: 10.14198/jhse.2017.124.20

Antunes, D., Rossato, M., Kons, R. L., Sakugawa, R. L., \& Fischer, G. (2017). Neuromuscular features in sprinters with cerebral palsy: case studies based on paralympic classification. J Exerc Rehabil, 13(6), 716-721. doi: 10.12965/jer.1735112.556

Bartolomeu, R. F., Costa, M. J., \& Barbosa, T. M. (2018). Contribution of limbs' actions to the four competitive swimming strokes: a nonlinear approach. J Sports Sci, 1-10. doi: $10.1080 / 02640414.2018 .1423608$

Beckman, E. M., Connick, M. J., \& Tweedy, S. M. (2016). How much does lower body strength impact Paralympic running performance? Eur J Sport Sci, 16(6), 669-676. doi: $10.1080 / 17461391.2015 .1132775$

Beckman, E. M., Connick, M. J., \& Tweedy, S. M. (2017). Assessing muscle strength for the purpose of classification in Paralympic sport: A review and recommendations. J Sci Med Sport, 20(4), 391-396. doi: 10.1016/j.jsams.2016.08.010 
Beckman, E. M., Newcombe, P., Vanlandewijck, Y., Connick, M. J., \& Tweedy, S. M. (2014). Novel strength test battery to permit evidence-based paralympic classification. Medicine (Baltimore), 93(4), e31. doi: 10.1097/MD.0000000000000031

Bohannon, R. W. (2005). Manual muscle testing: does it meet the standards of an adequate screening test? Clin Rehabil, 19(6), 662-667. doi: 10.1191/0269215505cr873oa

Burkett, B., Connick, M., Sayers, M., Hogarth, L., Stevens, T., Hurkx, M., \& Tweedy, S. (2017). Kinematic analyses of seated throwing activities with and without an assistive pole. Sports Engineering, 20(2), 163-170. doi: 10.1007/s12283-016-0221-y

Burkett, B., Payton, C., Van de Vliet, P., Jarvis, H., Daly, D., Mehrkuehler, C., Kilian, M., \& Hogarth, L. (2018). Performance characteristics of para Swimmers: How effective is the swimming classification system? Phys Med Rehabil Clin N Am, 29(2), 333-346. doi: 10.1016/j.pmr.2018.01.011

Connaboy, C., Naemi, R., Brown, S., Psycharakis, S., McCabe, C., Coleman, S., \& Sanders, R. (2016). The key kinematic determinants of undulatory underwater swimming at maximal velocity. J Sports Sci, 34(11), 1036-1043. doi: $10.1080 / 02640414.2015 .1088162$

Connick, M. J., Beckman, E., Deuble, R., \& Tweedy, S. M. (2016). Developing tests of impaired coordination for Paralympic classification: normative values and testretest reliability. Sports Engineering, 19(3), 147-154. doi: 10.1007/s12283-0160199-5

Connick, M. J., Beckman, E., Vanlandewijck, Y., Malone, L. A., Blomqvist, S., \& Tweedy, S. M. (2017). Cluster analysis of novel isometric strength measures produces a valid and evidence-based classification structure for wheelchair track racing. Br J Sports Med. doi: 10.1136/bjsports-2017-097558 
478 Cormie, P., McGuigan, M. R., \& Newton, R. U. (2011). Developing maximal neuromuscular power: part 2 - training considerations for improving maximal power production. Sports Med, 41(2), 125-146. doi: 10.2165/11538500-000000000-00000

Crowley, E., Harrison, A. J., \& Lyons, M. (2017). The Impact of Resistance Training on Swimming Performance: A Systematic Review. Sports Med. doi: 10.1007/s40279017-0730-2

Daly, D. J., \& Vanlandewijck, Y. (1999). Some criteria for evaluating the "fairness" of swimming classification. Adapted Physical Activity Quarterly, 16(3), 271-289

Delgado, M. R., \& Albright, A. L. (2003). Movement disorders in children: definitions, classifications, and grading systems. J Child Neurol, 18 Suppl 1, S1-8. doi: $10.1177 / 088307380301800150301$ doi: $10.1055 / \mathrm{s}-0033-1364023$

Dingley, A. A., Pyne, D. B., Youngson, J., \& Burkett, B. (2015). Effectiveness of a dry-land resistance training program on strength, power, and swimming performance in paralympic swimmers. J Strength Cond Res, 29(3), 619-626. doi: $10.1519 / J S C .0000000000000684$

Hogarth, L., Payton, C., Van de Vliet, P., Connick, M., \& Burkett, B. (2018). A novel method to guide classification of para swimmers with limb deficiency. Scand J Sci Med Sports, O(0). 1-10. doi: 10.1111/sms.13229

499 Hopkins, W. G. (2000). Measures of reliability in sports medicine and science. Sports Medicine, 30(1), 1-15. doi: Doi 10.2165/00007256-200030010-00001 
Hyde, A., Hogarth, L., Sayers, M., Beckman, E., Connick, M. J., Tweedy, S., \& Burkett, B. (2017). The Impact of an Assistive Pole, Seat Configuration, and Strength in Paralympic Seated Throwing. Int J Sports Physiol Perform, 12(7), 977-983. doi: 10.1123/ijspp.2016-0340

International Paralympic Committee. (2015). IPC Athlete Classification Code. Retrieved from https://www.paralympic.org/classification/2015-athlete-classification-code International Paralympic Committee. (2017). World Para Swimming Classification Rules and Regulations: International Paralympic Committee.

Jones, J. V., Pyne, D. B., Haff, G. G., \& Newton, R. U. (2018). Comparison of ballistic and strength training on swimming turn and dry-land leg extensor characteristics in elite swimmers. International Journal of Sports Science \& Coaching, 13(2), 262-269. doi: $10.1177 / 1747954117726017$

Liaw, A., \& Wiener, M. (2002). Classification and Regression by randomForest. R News, 2(3), $18-22$.

Loturco, I., Barbosa, A. C., Nocentini, R. K., Pereira, L. A., Kobal, R., Kitamura, K., . . . Nakamura, F. Y. (2016). A Correlational Analysis of Tethered Swimming, Swim Sprint Performance and Dry-land Power Assessments. Int J Sports Med, 37(3), 211218. doi: $10.1055 / \mathrm{s}-0035-1559694$

Martens, J., Figueiredo, P., \& Daly, D. (2015). Electromyography in the four competitive swimming strokes: a systematic review. J Electromyogr Kinesiol, 25(2), 273-291. doi: 10.1016/j.jelekin.2014.12.003

McGuigan, M. R., Newton, M. J., Winchester, J. B., \& Nelson, A. G. (2010). Relationship between isometric and dynamic strength in recreationally trained men. J Strength Cond Res, 24(9), 2570-2573. doi: 10.1519/JSC.0b013e3181ecd381 
Morouço, P. G., Marinho, D. A., Amaro, N. M., Pérez-Turpin, J. A., \& Marques, M. C. (2012). Effects of dry-land strength training on swimming performance: a brief review. Journal of Human Sport and Exercise, 7(2), 553-559. doi: 10.4100/jhse.2012.72.18

Morouco, P. G., Marinho, D. A., Izquierdo, M., Neiva, H., \& Marques, M. C. (2015). Relative Contribution of Arms and Legs in 30 s Fully Tethered Front Crawl Swimming. Biomed Res Int, 2015, 563206. doi: 10.1155/2015/563206

Morouco, P., Neiva, H., Gonzalez-Badillo, J. J., Garrido, N., Marinho, D. A., \& Marques, M. C. (2011). Associations between dry land strength and power measurements with swimming performance in elite athletes: a pilot study. J Hum Kinet, 29A, 105-112. doi: 10.2478/v10078-011-0065-2

Mukaka, M. M. (2012). A guide to appropriate use of correlation coefficient in medical research. Malawi Medical Journal, 24, 69-71.

Nicholson, V., Spathis, J., Hogarth, L., Connick, M., Beckman, E., Tweedy, S., . . Burkett, B. Establishing the reliability of a novel battery of range of motion tests to enable evidence-based classification In Para Swimming. Physical Therapy in Sport. doi: 10.1016/j.ptsp.2018.04.021

Oh, Y. T., Burkett, B., Osborough, C., Formosa, D., \& Payton, C. (2013). London 2012 Paralympic swimming: passive drag and the classification system. Br J Sports Med, 47(13), 838-843. doi: 10.1136/bjsports-2013-092192

Prins, J., \& Murata, N. (2008). Kinematic Analysis of Swimmers with Permanent Physical Disabilities. International Journal of Aquatic Research and Education, 4(6), 330-345. doi: $10.25035 /$ ijare.02.04.06

Psycharakis, S. G., \& Sanders, R. H. (2010). Body roll in swimming: a review. J Sports Sci, 28(3), 229-236. doi: 10.1080/02640410903508847 
Rogmann, J. J. (2013). Ordinal Dominance Statistics (orddom): An R Project for Statistical Computing package to compute ordinal, nonparametric alternatives to mean comparison (Version 3.1). Retrieved from http://cran.r-project.org/

Samson, M., Monnet, T., Bernard, A., Lacouture, P., \& David, L. (2015). Kinematic hand parameters in front crawl at different paces of swimming. J Biomech, 48(14), 37433750. doi: 10.1016/j.jbiomech.2015.07.034

Tweedy, S. M., Beckman, E. M., \& Connick, M. J. (2014). Paralympic classification: conceptual basis, current methods, and research update. $P M R, 6(8$ Suppl), S11-17. doi: 10.1016/j.pmrj.2014.04.013

Tweedy, S. M., Mann, D., \& Vanlandewijck, Y. C. (2016). Research needs for the development of evidence-based systems of classification for physical, vision, and intellectual impairments. In Y. C. Vanlandewijck \& W. R. Thompson (Eds.), Training and Coaching the Paralympic Athlete (pp. 122-149): John Wiley \& Sons, Ltd.

Tweedy, S. M., \& Vanlandewijck, Y. C. (2011). International Paralympic Committee position stand--background and scientific principles of classification in Paralympic sport. Br J Sports Med, 45(4), 259-269. doi: 10.1136/bjsm.2009.065060

Woods, C. T., Veale, J., Fransen, J., Robertson, S., \& Collier, N. F. (2018). Classification of playing position in elite junior Australian football using technical skill indicators. J Sports Sci, 36(1), 97-103. doi: 10.1080/02640414.2017.1282621

Wu, S. K., \& Williams, T. (1999). Paralympic swimming performance, impairment, and the functional classification system. Adapted Physical Activity Quarterly, 16(3), 251-270 
Figure captions
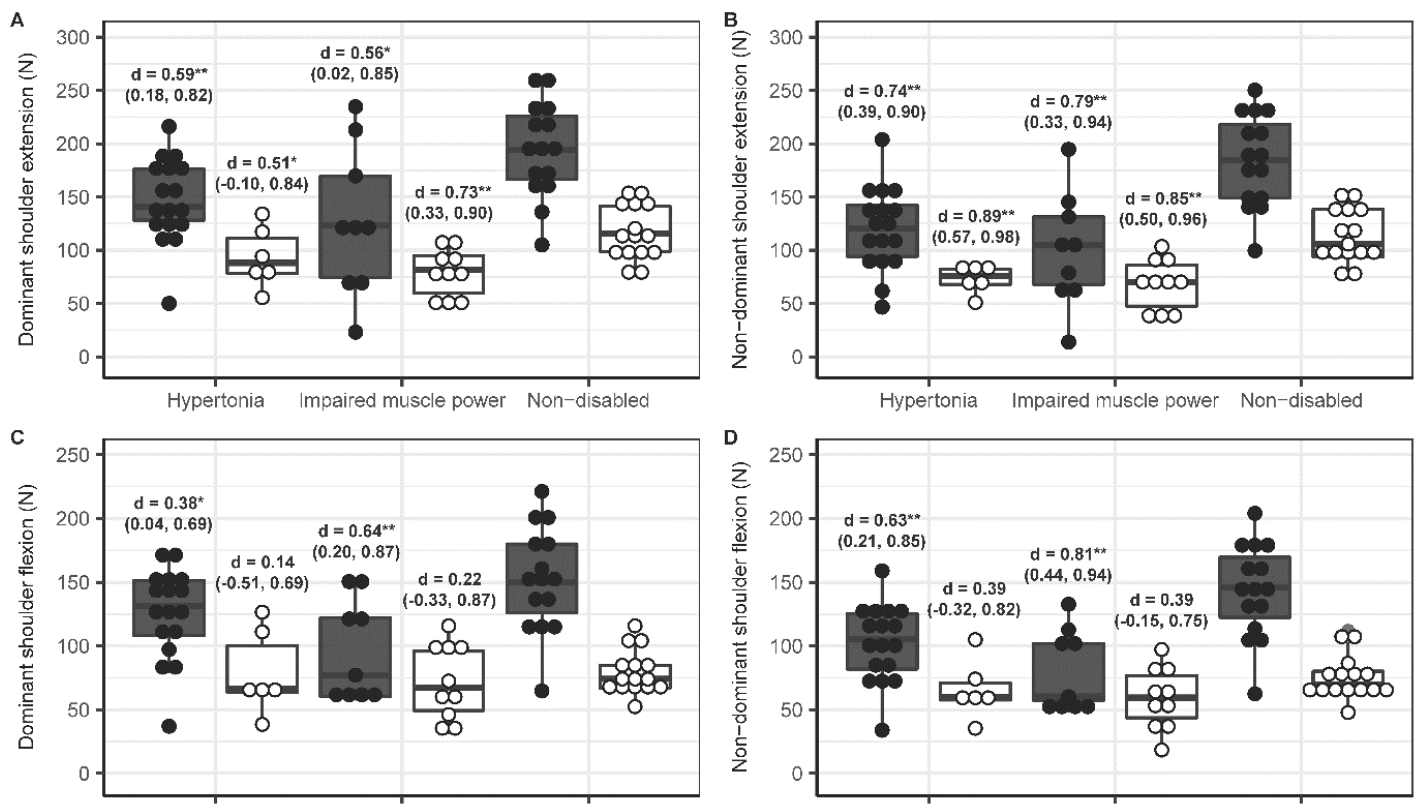

Hypertonia Impaired muscle power Non-disabled

Hypertonia Impaired muscle power Non-disabled
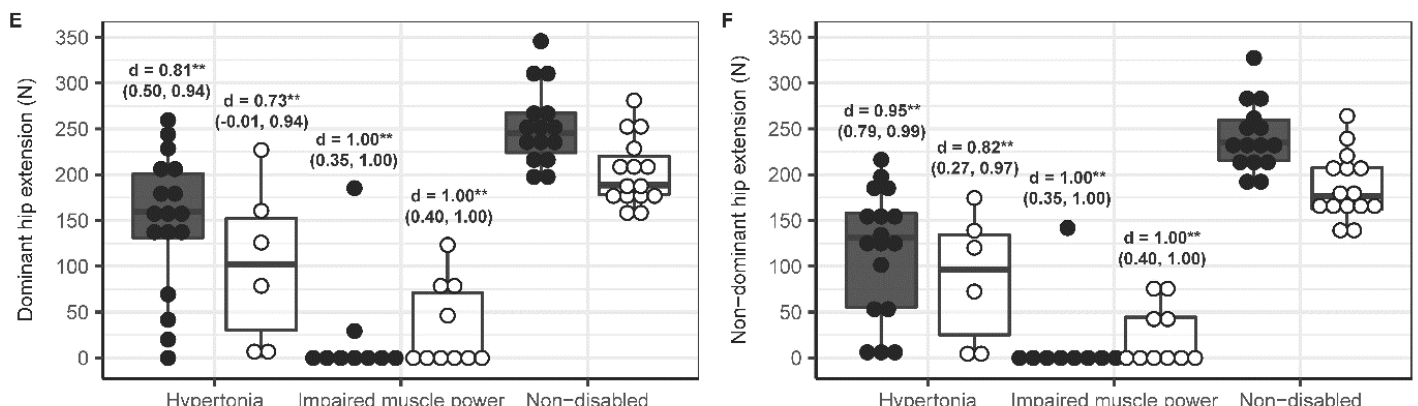

Hypertonia Impaired muscle power Non-disabled

Hypertonia Impaired muscle power Non-disabled
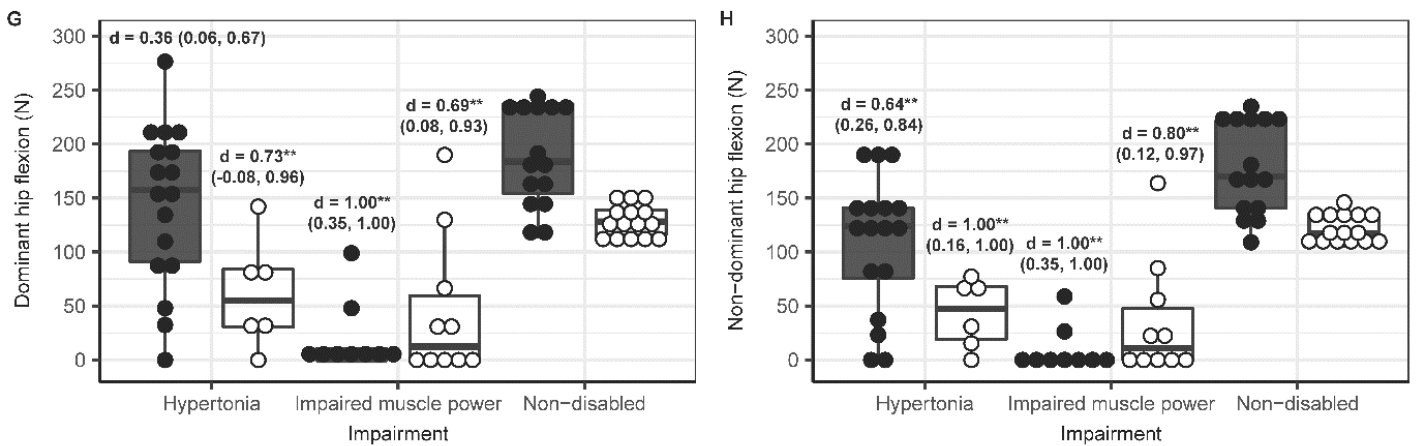

Figure 1. Strength test scores for Para swimmers with physical impairments and non-disabled participants. Scores a for (A) dominant and (B) non-dominant shoulder extension, (C) dominant and (D) non-dominant shoulder flexion, (E) dominant and (F) non-dominant hip extension, and (G) dominant and $(\mathrm{H})$ non-dominant hip flexion. Data are reported for male (dark colour box plots) and female (white colour box plots) participants. Data are Cliff's delta scores with $95 \% \mathrm{Cl}$ indicating differences between para swimmers and non-disabled participants. $*(p \leq 0.05)$ and ${ }^{* *}(p \leq 0.01)$ indicate significance. 
A

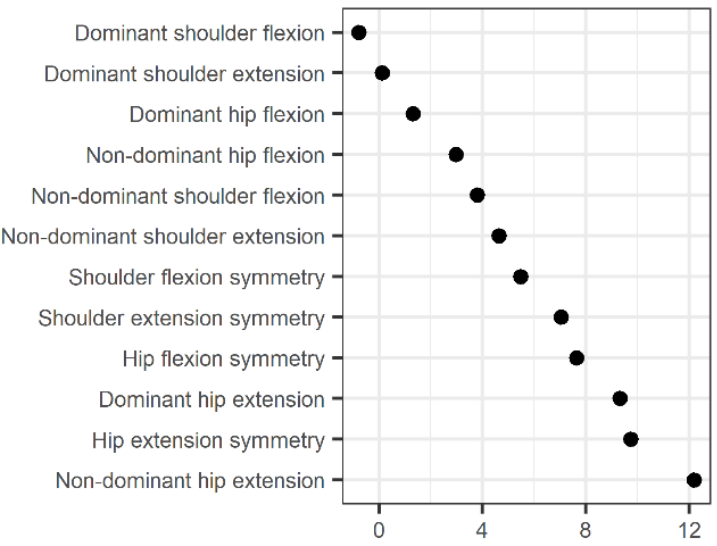

B

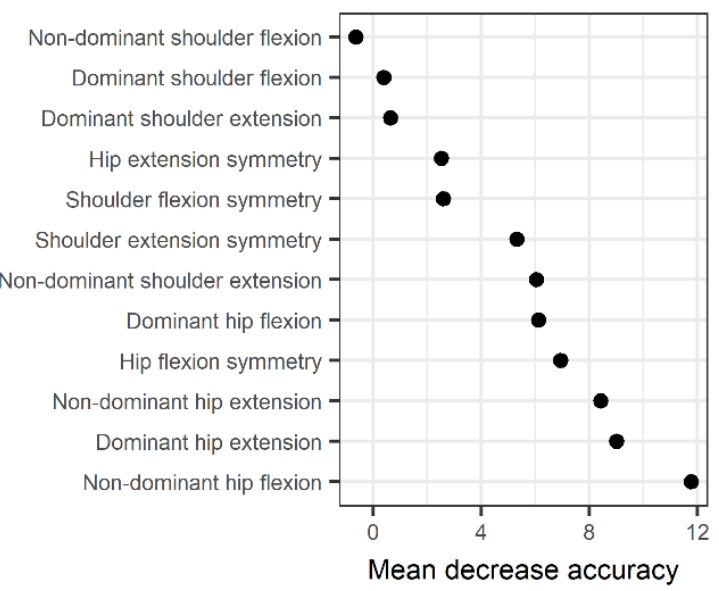

Figure 2. Mean decrease in accuracy scores indicating variable importance in classifying participants with and without physical impairment. Scores are reported for (A) male and (B) female participants. The variable importance score is the decrease in accuracy for each predictor variable when it is excluded from the classification model. The plot shows "non-dominant hip flexion" strength to be the strongest predictor in whether male and female participants did or did not have physical impairment. 
A

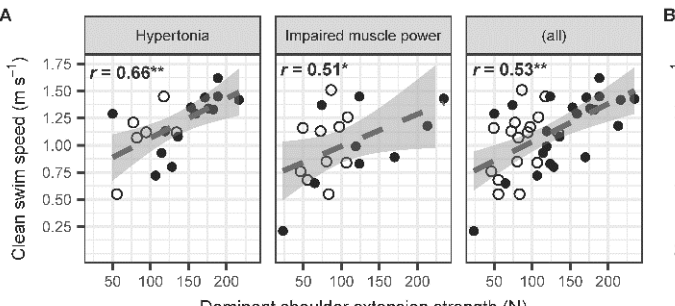

Dominant shoulder extension strength $(\mathrm{N})$

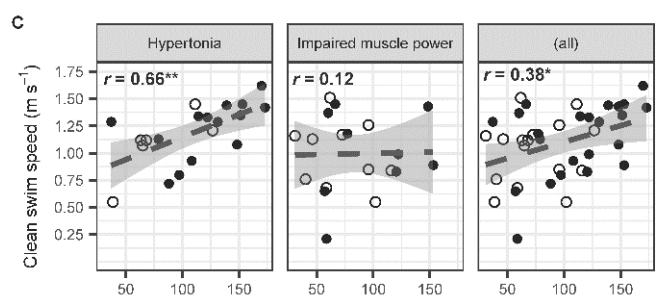

Dominant shoulder flexion strength $(\mathrm{N})$

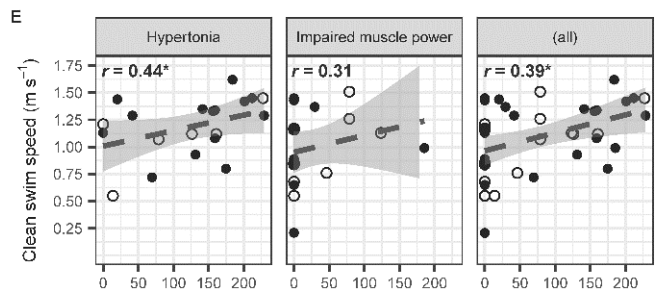

Dominant hip extension strength (N)

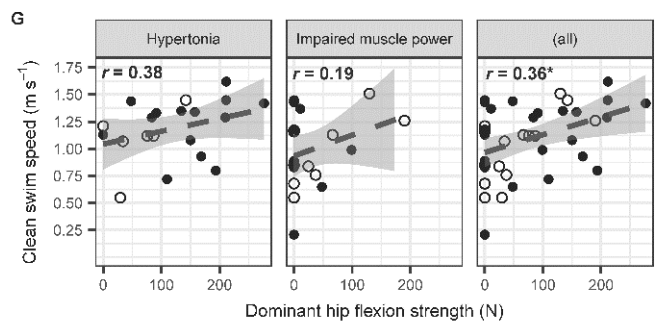

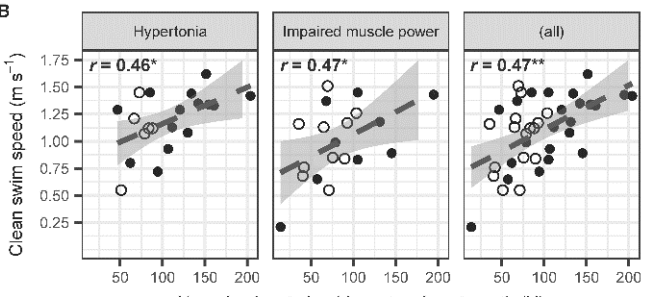

Non-dominant shoulder extension strength $(\mathrm{N})$

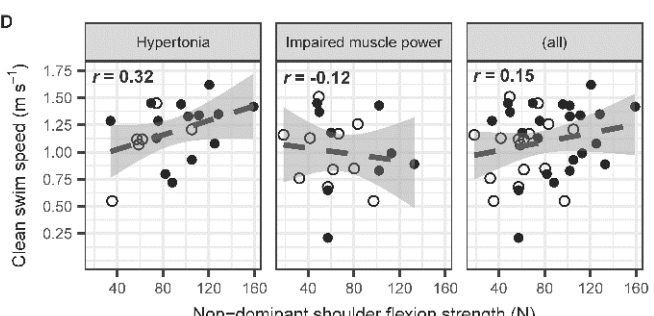

Non-dominant shoulder flexion strength (N)

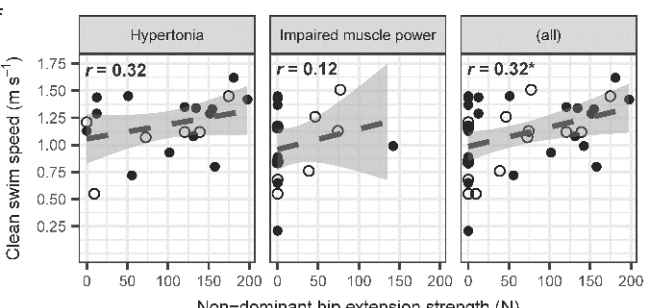

Non-dominant hip extension strength $(\mathrm{N})$

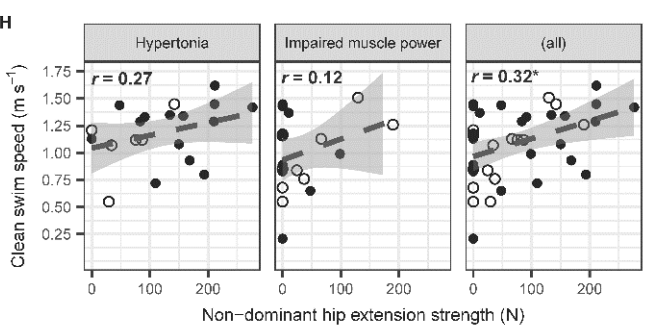

Figure 3. Strength of association between strength test scores and clean swim speed during maximal freestyle swimming. Data are Spearman correlation coefficients indicating strength of association between clean swim speed and (A) dominant and (B) non-dominant shoulder extension, (C) dominant and (D) don-dominant shoulder flexion, (E) dominant and (F) non-dominant hip extension, and $(G)$ dominant and $(H)$ non-dominant hip flexion. Plots show these associations for the combined cohort of para swimmers $(n=39)$ and independently for Para swimmers with hypertonia $(n=20)$ or impaired muscle power $(n=19)$. Male (dark colour dots) and female (white colour dots) participants were pooled for analysis. ${ }^{*}(p \leq 0.05)$ and ${ }^{* *}(p \leq 0.01)$ indicate significance. 
Table 1. Characteristics of non-disabled participants and para swimmers with physical impairment.

\begin{tabular}{|c|c|c|c|c|}
\hline & & Hypertonia & Impaired muscle power & Non-disabled \\
\hline & Males & $\mathrm{n}=17$ & $n=9$ & $\mathrm{n}=15$ \\
\hline & Females & $n=6$ & $n=10$ & $\mathrm{n}=15$ \\
\hline \multirow[t]{2}{*}{ Age (yrs) } & Males & $26.5(7.0)$ & $31.5(7.7)$ & $24(4)$ \\
\hline & Females & $19.8(4.1)$ & $29.9(10.2)$ & $23(5)$ \\
\hline \multirow[t]{2}{*}{ Body mass (kg) } & Males & $67.9(9.8)$ & $63.4(14.4)$ & $79.8(11.4)$ \\
\hline & Females & $58.2(10.30$ & $56.2(10.3)$ & $68.1(9.7)$ \\
\hline \multirow[t]{2}{*}{ Stature $(\mathrm{cm})$} & Males & $172.0(8.8)$ & $167.2(12.4)$ & $182.7(7.7)$ \\
\hline & Females & $160.7(9.0)$ & $153.0(13.4)$ & $171.4(7.0)$ \\
\hline Reported exercise & & Median $=7.5$ & Median $=7$ & Median $=6$ \\
\hline $\begin{array}{l}\text { frequency } \\
\text { (n/week) }\end{array}$ & & Range $=2$ to 15 & Range $=3$ to 14 & Range $=3$ to 14 \\
\hline Accumulated & & Median $=720$ & Median $=630$ & Median $=360$ \\
\hline $\begin{array}{l}\text { exercise duration } \\
\text { ( } \mathrm{min} / \text { week) }\end{array}$ & & Range $=180$ to 1200 & Range $=180$ to 1170 & Range $=150$ to 1200 \\
\hline \multirow[t]{7}{*}{$\begin{array}{l}\text { Reported } \\
\text { activities }\end{array}$} & & $\begin{array}{l}\text { Competitive swimming } \\
(n=23)\end{array}$ & $\begin{array}{l}\text { Competitive swimming } \\
(n=19)\end{array}$ & $\begin{array}{l}\text { Resistance training } \\
(n=17)\end{array}$ \\
\hline & & Resistance training & Resistance training $(n=6)$ & Recreational fitness ${ }^{a}$ \\
\hline & & $(n=15)$ & Wheelchair rugby $(n=1)$ & $(n=13)$ \\
\hline & & & Pilates and Yoga $(n=1)$ & Competitive sport ${ }^{b}$ \\
\hline & & & & $(n=12)$ \\
\hline & & & & Recreational sport ${ }^{\mathrm{c}}(\mathrm{n}=8)$ \\
\hline & & & & Pilates and Yoga $(n=4)$ \\
\hline Competitive & & International $\left.\right|^{d}(n=9)$ & International ${ }^{d}(n=9)$ & \\
\hline standard & & National $(n=14)$ & National $(n=10)$ & \\
\hline Competitive swim & & Median $=9.5$ & Median $=7$ & \\
\hline experience (yrs) & & Range $=2$ to 26 & Range $=4$ to 20 & \\
\hline \multirow[t]{8}{*}{ S Class } & & S3 $(n=1)$ & $S 1(n=2)$ & \\
\hline & & S4 $(n=4)$ & S3 $(n=2)$ & \\
\hline & & S5 $(n=2)$ & S4 $(n=2)$ & \\
\hline & & $S 6(n=5)$ & S5 $(n=3)$ & \\
\hline & & $S 7(n=2)$ & $S 6(n=3)$ & \\
\hline & & S8 $(n=7)$ & $S 7(n=2)$ & \\
\hline & & S9 $(n=2)$ & S8 $(n=3)$ & \\
\hline & & & $59(n=2)$ & \\
\hline Medical & & Diplegic CP (n=8) & Incomplete $\mathrm{SCl}(\mathrm{n}=4)$ & \\
\hline \multirow[t]{6}{*}{ conditions } & & Hemiplegic CP (n=9) & Complete $\mathrm{SCl}(\mathrm{n}=8)$ & \\
\hline & & Quadriplegic CP $(n=4)$ & Charcot-Marie-Tooth & \\
\hline & & Other $(n=2)$ & disease $(n=2)$ & \\
\hline & & & Spina bifida (2) & \\
\hline & & & Polio $(n=1)$ & \\
\hline & & & Other $(n=3)$ & \\
\hline
\end{tabular}

$\overline{\mathrm{CP}}=$ cerebral palsy, $\mathrm{SCl}=$ spinal cord injury. $\mathrm{S}$ Class = para swimmers' current class for freestyle, backstroke and butterfly swimming events. ${ }^{a}$ Reported recreational fitness activities included moderate to high-intensity aerobic exercise, and group fitness classes. ${ }^{b}$ Reported competitive sports training or competition included athletics, rugby, AFL, football, powerlifting and swimming. ${ }^{~}$ Reported recreational sport competition included football, badminton, netball, jujitsu, dance and surfing. ${ }^{d}$ Para swimmers were classified as international standard if they had competed during a Paralympic or World Championship event. 
Table 2. Reliability of strength test measures in non-disabled participants.

\begin{tabular}{|c|c|c|c|c|c|c|c|}
\hline & & $\begin{array}{c}\text { Trial } 1 \\
\text { Mean (SD) } \\
(\mathrm{N})\end{array}$ & $\begin{array}{c}\text { Trial } 2 \\
\text { Mean (SD) } \\
(\mathrm{N})\end{array}$ & $\begin{array}{c}\Delta \mathrm{T} 2-\mathrm{T} 1 \\
\text { Mean (SD) } \\
(\mathrm{N})\end{array}$ & $\begin{array}{c}\text { SEM } \\
(\mathrm{N})\end{array}$ & $\begin{array}{l}\text { CV } \\
(\%)\end{array}$ & $\begin{array}{c}\text { ICC } \\
(95 \% \mathrm{Cl})\end{array}$ \\
\hline \multirow[t]{2}{*}{$\begin{array}{l}\text { Shoulder extension } \\
\text { strength }\end{array}$} & Dominant & $170.3(54.0)$ & $172.0(52.1)$ & $1.7(15.1)$ & 10.2 & 6.9 & $\begin{array}{c}0.97(0.92- \\
0.99)\end{array}$ \\
\hline & $\begin{array}{l}\text { Non- } \\
\text { dominant }\end{array}$ & $156.0(50.4)$ & $157.8(44.3)$ & $1.9(18.3)$ & 12.9 & 8.5 & $\begin{array}{c}0.94(0.87- \\
0.98)\end{array}$ \\
\hline \multirow[t]{2}{*}{$\begin{array}{l}\text { Shoulder flexion } \\
\text { strength }\end{array}$} & Dominant & $128.1(39.3)$ & $122.3(35.6)$ & $-5.9(12.1)$ & 8.6 & 6.8 & $\begin{array}{c}0.97(0.91- \\
0.99)\end{array}$ \\
\hline & $\begin{array}{l}\text { Non- } \\
\text { dominant }\end{array}$ & $117.1(37.0)$ & $110.4(33.4)$ & $-6.7(14.1)$ & 10.0 & 7.7 & $0.96(0.9-0.98)$ \\
\hline \multirow[t]{2}{*}{$\begin{array}{l}\text { Hip extension } \\
\text { strength }\end{array}$} & Dominant & $245.1(46.9)$ & $242.7(46.1)$ & $-2.5(20.3)$ & 14.4 & 6.4 & $\begin{array}{c}0.91(0.78- \\
0.96)\end{array}$ \\
\hline & $\begin{array}{l}\text { Non- } \\
\text { dominant }\end{array}$ & $225.3(38.3)$ & $222.7(45.4)$ & $-2.6(24.5)$ & 17.4 & 8.0 & $\begin{array}{c}0.85(0.65- \\
0.94)\end{array}$ \\
\hline \multirow[t]{2}{*}{ Hip flexion strength } & Dominant & $168.8(46.7)$ & $164.7(42.7)$ & $-4.2(19.7)$ & 14.0 & 9.1 & $\begin{array}{c}0.91(0.78- \\
0.96)\end{array}$ \\
\hline & $\begin{array}{l}\text { Non- } \\
\text { dominant }\end{array}$ & $157.2(43.5)$ & $155.5(45.7)$ & $-1.7(15.2)$ & 10.7 & 6.8 & $\begin{array}{c}0.95(0.89- \\
0.98)\end{array}$ \\
\hline
\end{tabular}

SEM = standard error of measurement, $\mathrm{CV}=$ coefficient of variation, $\mathrm{ICC}=$ intraclass correlation coefficient, $\mathrm{Cl}=$ confidence interval. 


\section{Supplementary online material}

Supplementary Table 1. Description of isometric strength tests developed for Para swimming classification.

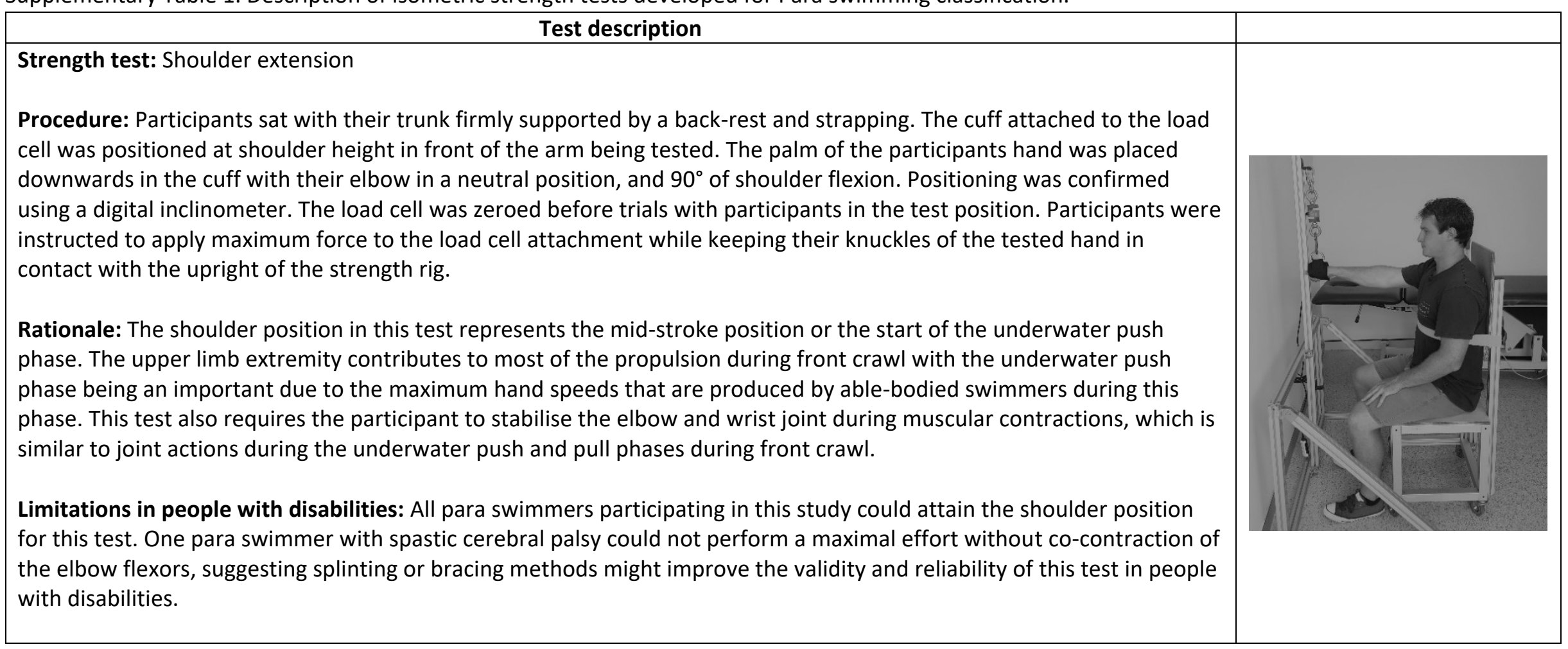


Supplementary Table 1 (continued). Description of isometric strength tests developed for Para swimming classification.

\begin{tabular}{|l|}
\hline Strength test: Shoulder flexion \\
Procedure: Participants sat with their trunk firmly supported by a back-rest and strapping. The hand of the tested arm \\
was placed in the cuff with the palm facing forward in a neutral position, elbow in a neutral position, and shoulder in a \\
neutral. The load cell was attached to the strength rig at the height of the tested hand with the attachment taut when \\
participants were in the test position. The load cell was zeroed before trials with participants shoulder in an extended \\
position so that the load cell attachment was not taut. Participants were instructed to "take the slack" of the load cell \\
attachment and pause prior to commencing the maximal effort test. \\
Rationale: Although being dissimilar to front crawl kinematics in able-bodied swimmers, this test was included in the \\
battery to explain activity limitation in para swimmers with severe physical impairments. Some of these para swimmers \\
will use modified stroke patterns in the freestyle discipline, such as double armed backstroke. The shoulder positioning \\
during this test might represent part of the underwater propulsion phase for these swimmers. \\
Limitations in people with disabilities: While all para swimmers could attain the shoulder positioning for this test, one \\
para swimmer with spastic cerebral palsy could not perform the shoulder action without co-contraction of the elbow \\
flexors, suggesting splinting or bracing methods might improve the validity and reliability of this test in people with \\
disabilities.
\end{tabular}


Supplementary Table 1 (continued). Description of isometric strength tests developed for Para swimming classification.

Strength test: Hip extension
Procedure: Participants were in a supine position on a massage plinth with their legs off the bench at the popliteal
crease. The tested leg was placed in an ankle cuff that attached the load cell to the strength rig so that the tested leg was
in $15^{\circ}$ of hip flexion, and neutral knee and ankle positioning. Arms were folded across the chest, and the foot of the
tested leg was not in contact with the strength rig. Positioning was confirmed using a digital inclinometer that was
placed on the mid-section of the thigh. The load cell was zeroed before trials with participants in the test position.
Rationale: The leg kick contributes to propulsion and plays an important role in stabilising the body in coordination with
the arm stroke during front crawl swimming. This test was designed to explain the contribution of the posterior chain in
allowing para swimmers to maintain streamlined body positioning during freestyle. Hip extension strength is also
important to starts and turns performance.
Limitations in people with disabilities: Several para swimmers with contractures around the hip and knee could not
achieve standardised positioning for this test. Para swimmers with diplegic cerebral palsy often could not achieve full
knee extension. One para swimmer with severe hip and knee contractures had to perform the test in a modified seated
position with the ankle strap attached as the lower thigh.


Supplementary Table 1 (continued). Description of isometric strength tests developed for Para swimming classification.

\begin{tabular}{l}
\hline Strength test: Hip flexion \\
Procedure: Participants were in a supine position on a massage plinth with their legs off the bench at the popliteal \\
crease. The tested leg was placed in an ankle cuff. The load cell attachment was positioned so that it was taut when the \\
hip and knee of the tested leg were horizontal. Arms were folded across the chest, and the foot of the tested leg was not \\
in contact with the strength rig. The load cell was zeroed before trials with participants leg in a relaxed position so that \\
the load cell attachment was not taut. Participants were instructed to "take the slack" of the load cell attachment and \\
pause prior to commencing the maximal effort test. \\
Rationale: The leg kick contributes to propulsion and plays an important role in stabilising the body in coordination with \\
the arm stroke during front crawl swimming. This test was designed to explain the contribution of kip and knee flexion to \\
the drag and lift forces generated by the leg kick during front crawl. \\
Limitations in people with disabilities: Several para swimmers with contractures around the hip and knee could not \\
achieve standardised positioning for this test. Para swimmers with diplegic cerebral palsy often could not achieve full \\
knee extension. One para swimmer with severe hip and knee contractures had to perform the test in a modified seated \\
position with the ankle strap attached as the lower thigh.
\end{tabular}


Supplementary online material

Supplementary Table 2. Strength test measures (mean \pm SD) in non-disabled participants.

\begin{tabular}{llcc}
\hline & & Males $(\mathrm{n}=15)$ & Females $(\mathrm{n}=15)$ \\
\hline Shoulder extension strength & Dominant $(\mathrm{N})$ & $194.3(44.2)$ & $117.0(25.6)^{*}$ \\
& Non-dominant $(\mathrm{N})$ & $184.7(43.0)$ & $113.4(25.3)^{*}$ \\
& Symmetry index & $0.95(0.03)$ & $0.97(0.02)$ \\
Shoulder flexion strength & Dominant (N) & $152.3(40.8)$ & $79.2(17.6)^{*}$ \\
& Non-dominant (N) & $142.9(36.5)$ & $74.0(16.6)^{*}$ \\
& Symmetry index & $0.94(0.03)$ & $0.94(0.05)$ \\
& & & \\
Hip extension strength & Dominant (N) & $252.8(42.8)$ & $192.7(34.6)^{*}$ \\
& Non-dominant (N) & $240.8(37.3)$ & $187.1(36.0)^{*}$ \\
& Symmetry index & $0.95(0.02)$ & $0.92(0.05)$ \\
Hip flexion strength & & & $128.8(14.9)^{*}$ \\
& Dominant (N) & $188.6(46.2)$ & $122.3(14.0)^{*}$ \\
& Non-dominant (N) & $178.7(43.1)$ & $0.95(0.04)$ \\
\hline
\end{tabular}

* indicates significant difference $(p<0.01)$ to male group. 